\title{
Perfil dos portadores de cavidade anoftálmica - estudo na Faculdade de Medicina de Botucatu - UNESP
}

\author{
Profile of patients with anophthalmic cavity - study at Faculdade de Medicina de Botucatu - UNESP
}

Silvia Narikawa ${ }^{1}$, Kryscia Leiko Natsuaki ${ }^{1}$, Juliana Fruet ${ }^{2}$, Carlos Roberto Padovani ${ }^{3}$, Silvana Artioli Schellini ${ }^{4}$

\section{RESUMO}

Objetivo: Avaliar o perfil dos portadores de cavidade anoftálmica e a evolução dos pacientes com os tratamentos empregados.

Métodos: Estudo retrospectivo, realizado na Faculdade de Medicina de Botucatu, avaliando-se o perfil e a evolução dos portadores de cavidade anoftálmica, tratados no serviço. Os dados foram estudados segundo frequência de ocorrência e análise de associação.

Resultados: Os portadores de cavidade anoftálmica eram mais frequentemente em homens, trabalhadores em atividades exercidas com as mãos, jovens. O trauma e a phthisis bulbi foram as causas mais encontradas, havendo diferenças de acordo com a faixa etária e sexo. O índice complicações foi alto (57,1\% dos casos), a maioria ocorrendo tardiamente.

Conclusões: $O$ trauma continua sendo causa importante de perda do olho. As complicações que ocorrem durante o acompanhamento do portador de cavidade anoftálmica são frequentes e ocorrem, em geral, tardiamente, o que reforça a necessidade de acompanhamento periódico e prolongado destes pacientes.

Descritores: Anoftalmia/cirurgia; Anoftalmia/complicações; Seguimentos; Resultado de tratamento

\begin{abstract}
Purpose: To evaluate the profile of anophthalmic cavity carriers and patients' evolution with the employed treatments.

Methods: Retrospective study carried out at Faculdade de Medicina de Botucatu, to evaluate the profile and the evolution of patients with anophthalmic cavity treated in our service. Data were evaluated according to the frequency of occurrence and analysis of association.

Results: Anophthalmic cavity was more frequently observed in young males, workers in activities carried on with their hands. Trauma and phthisis bulbi were the most frequent causes, with differences according to age and gender. The complications were high ( $57.1 \%$ of cases), the majority occurred belatedly.

Conclusions: Trauma is still an important cause of loss of the eye. The complications during the follow-up of the patient are frequent, occurring in general, very late, which reinforces theneed for periodic and prolonged monitoring of patients with anophthalmic cavity.
\end{abstract}

Keywords: Anophthalmos/surgery; Anophthalmos/complications; Follow-up studies; Treatment outcome

\section{INTRODUÇÃO}

A cavidade anoftálmica é uma condição frequente nos serviços especializados em plástica ocular, podendo ser o resultado de afecções congênitas ou adquiridas. O tratamento adequado dos portadores de cavidade anoftálmica tem sido um desafio para muitos profissionais, que lutam para devolver pelo menos a aparência, já que a função está perdida em definitivo ${ }^{(1-3)}$.

A cavidade anoftálmica congênita é uma anomalia rara. Pode ser unilateral ou bilateral e a severidade das deformidades é variável. A malformação pode ter diferentes graus, indo desde a anoftalmia clínica, quando existe um olho microftálmico, muitas vezes não detectado no exame clínico de rotina, até a ausência do bulbo ocular que ocorre devido à falha completa na formação da vesícula óptica, chamada de anoftalmia verdadeira(4).

A falta do bulbo ocular ou o bulbo microftálmico levam ao não desenvolvimento da órbita, resultando em assimetria facial. Nesses pacientes, o tratamento tem como objetivo primordial estimular o crescimento adequado da órbita, assim como expandir a cavidade ${ }^{(1,2,5)}$.

A cavidade anoftálmica adquirida pode ser resultado de diversas causas como trauma, glaucoma, tumor, endoftalmite, entre outros. Estudo realizado na Faculdade de Medicina de Botucatu, no período entre 1990 e 1998, mostrou que naquela região as causas mais frequentes de cavidade anoftálmica adquirida eram a phthisis bulbi, endoftalmite e trauma(6).

É importante que o portador de anoftalmia seja reabilitado para conseguir retornar às suas atividades cotidianas e sociais. A cirurgia, quando necessária, deve ser realizada visando sempre a melhor condição para adaptação de uma prótese externa, que proporcione uma aparência cosmética aceitável e a preservação da função das pálpebras ${ }^{(1)}$.

A enucleação e a evisceração são as técnicas utilizadas para a remoção do bulbo ocular ou do seu conteúdo. A longo prazo, o portador de cavidade anoftálmica desenvolve enoftalmo, aumento

${ }^{1}$ Physician, Departamento de Oftalmologia, Otorrinolaringologia e Cirurgia de Cabeça e Pescoço Faculdade de Medicina de Botucatu, Universidade Estadual Paulista "Julio de Mesquita Filho" UNESP - Botucatu (SP), Brazil.

2 Student, Faculdade de Medicina de Botucatu, Universidade Estadual Paulista "Julio de Mesquita Filho" - UNESP - Botucatu (SP), Brazil.

3 Professor, Departamento de Bioestatística, Instituto de Biociências de Botucatu, Universidade Estadual Paulista "Julio de Mesquita Filho" - UNESP - Botucatu (SP), Brazil.

4 Professor, Departamento de Oftalmologia, Otorrinolaringologia e Cirurgia de Cabeça e Pescoço, Faculdade de Medicina de Botucatu, Universidade Estadual Paulista "Julio de Mesquita Filho" UNESP - Botucatu (SP), Brazil.

Funding: No specific financial support was available for this study.

Disclosure of potential conflicts of interest: S.Narikawa, None; K.L.Natsuaki, None; J.Fruet, None; C.R.Padovani, None; S.A.Schellini, None.

Correspondence address: Silvana Artioli Schellini. Dep. OFT/ORL/CCP - Faculdade de Medicina de Botucatu - UNESP - Botucatu (SP) - 18618-970 - Brazil - E-mail: sartioli@fmb.unesp.br 
da profundidade e elevação do sulco palpebral superior, ptose e flacidez da pálpebra inferior caracterizando a síndrome da cavidade anoftálmica ${ }^{(2)}$. Portadores de cavidade anoftálmica, mesmo após as cirurgias reconstrutivas, podem apresentar alterações na aparência ${ }^{(1,7)}$.

Para a reconstrução dessas cavidades existem diversos tipos de implantes, lisos ou porosos, de diversos materiais, como os confeccionados em polimetilmetacrilato, silicone, polietileno, hidroxiapatita, vidro, entre outros. Podem necessitar de envoltório, podendo-se utilizar esclera doadora, fáscia lata ou periósteo(8).

A utilização dos diversos materiais, assim como a escolha dos procedimentos a serem realizados, dependem da disponibilidade e de alguns fatores relacionados ao próprio portador de cavidade anoftálmica. O resultado final também pode ser variado, conforme fatores relativos à causa da perda do olho ou de seu conteúdo, tipo de cirurgia efetuada e resposta do hospedeiro(9).

A remoção do bulbo ocular ou do seu conteúdo acarreta alterações na anatomia e na fisiologia da órbita, afetando a aparência, a função da cavidade e, principalmente, a mobilidade das pálpebras e da prótese (10) $^{(10}$

É bastante frequente encontrar indivíduos que sofrem contração da cavidade anoftálmica, com depressão da pálpebra superior e/ou retração da pálpebra inferior ${ }^{(11)}$.

E muitas complicações podem ocorrer durante o acompanhamento do paciente, sendo mais frequentemente relatadas a exposição, associada ou não a extrusão do implante, infecções, granuloma piogênico e secreção(8).

Estudos a respeito do perfil do portador de cavidade anoftálmica, assim como a resposta aos tratamentos realizados, poderiam informar quais seriam os pontos mais importantes a serem considerados para melhor tratar o paciente.

\section{OBJETIVO}

O objetivo deste estudo foi avaliar o perfil dos portadores de cavidade anoftálmica, assim como a evolução dos pacientes com os tratamentos empregados.

\section{MÉTODOS}

Este foi um estudo retrospectivo, os dados foram coletados a partir dos prontuários dos portadores de cavidade anoftálmica, atendidos no ambulatório de Plástica Ocular da Faculdade de Medicina de Botucatu - UNESP. O serviço funciona há 25 anos e o estudo procurou levantar os casos que chegaram ao serviço no período de 1988 a 2006, totalizando 167 casos dos quais foram excluídos os casos cujos prontuários apresentavam-se inativos e com informações incompletas. Assim, foram selecionados 107 casos para o presente estudo.

Os dados avaliados foram: profissão e sexo do paciente, causa da perda do olho, idade da perda do olho, acuidade visual no olho contralateral, tipo de cirurgia realizada, tipo de implante utilizado, complicações precoces (considerado até um ano da perda ocular) ou tardias, realização ou não de outros procedimentos, como enxertos de pele e dermoadiposo, aspecto estético e tempo de seguimento.

Com relação à profissão, os trabalhadores foram classificados de acordo com o tipo de tarefa que desempenhavam, levando-se em conta se o indivíduo utilizava principalmente a força física, se realizava trabalhos primariamente manuais ou se as atividades eram mais relacionadas ao intelecto. As crianças, os aposentados e desempregados foram separados em uma categoria à parte.

Os dados obtidos foram transferidos para Planilha Excel, tendo sido analisados estatisticamente segundo a frequência de ocorrência e testes de associação.

\section{RESULTADOS}

Após revisão, observou-se que 107 prontuários estavam adequados para inclusão no estudo. Desta forma, foram analisados dados de 107 pacientes, dos quais 75 eram homens e 32, mulheres. Houve uma frequência maior de ocorrência de perda do olho ou de seu conteúdo nas idades superiores a 40 anos $(31,9 \%)$ e entre 21 a 40 anos de idade (29,8\%). Os pacientes com idade menor que 10 anos corresponderam a $25,5 \%$ da amostra e o restante apresentavase entre 10 a 20 anos.

O trauma foi a causa mais encontrada (38,5\%), seguido de phthisis bulbi (17,7\%). A distribuição de acordo com o sexo apontou o trauma como causa mais frequente na população masculina e na feminina, phthisis bulbi (Gráfico 1).

Na população menor que 10 anos, encontrou-se uma frequência maior de tumores como causa principal. Já na população com idade entre 10 a 40 anos a causa mais encontrada foi o trauma; e nos mais velhos, com idade maior que 40 anos, a endoftalmite foi a mais frequente (Gráfico 2).

Com relação ao tipo de atividade, na população masculina a perda do olho ou de seu conteúdo estiveram mais associadas com os trabalhadores que utilizam principalmente a força física e as mãos (40,8\%); e, na feminina, uma ocorrência maior de profissionais classificados em outras categorias (51,9\%).

A grande maioria dos portadores de cavidade anoftálmica apresentou acuidade visual no olho contralateral entre 0,7 e 1,0 $(77,6 \%)$. Foi encontrada uma ocorrência de 9,2\% indivíduos que apresentavam visão subnormal no olho contralateral, ou seja, acuidade visual menor 0,3 no melhor olho. Os indivíduos que apresentavam acuidade visual no olho contralateral maior que 0,3 ti-

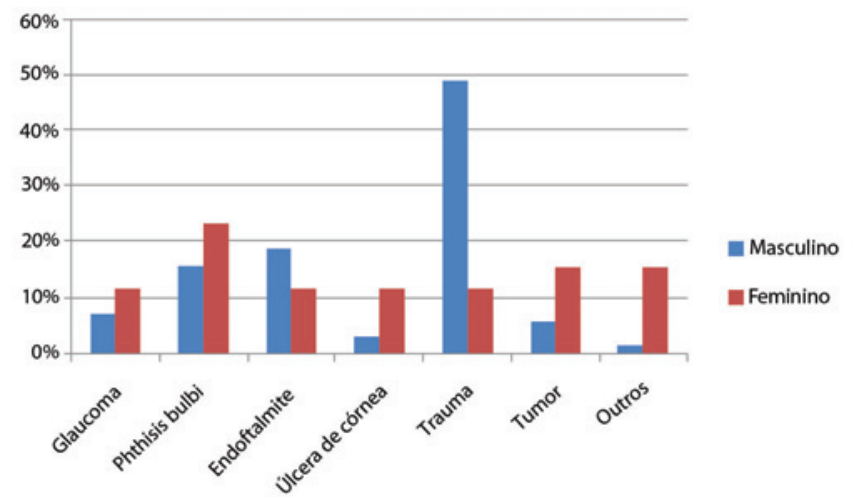

Gráfico 1. Distribuição das causas de perda do olho ou do seu conteúdo, segundo o sexo - UNESP, 2010.

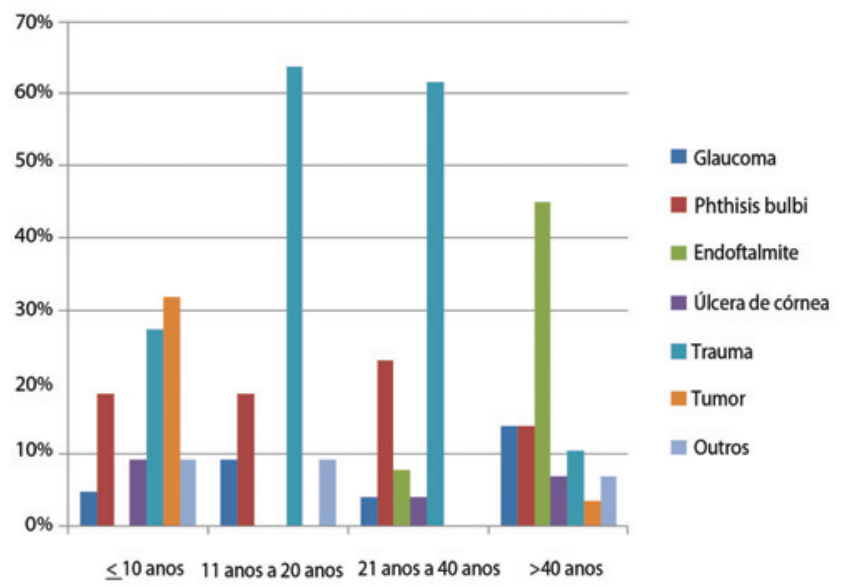

Gráfico 2. Distribuição das causas de perda do olho ou do seu conteúdo, segundo a idade da perda - UNESP, 2010. 
veram como principal causa de perda do olho o trauma $(43,8 \%)$ seguido pela phthisis bulbi $(17,2 \%)$ e endoftalmite (16,6\%). Já aqueles cuja acuidade visual no olho contralateral foi menor ou igual a 0,3 , apresentaram como principais causas de perda do olho o glaucoma $(33,3 \%)$ e a endoftalmite $(33,3 \%)$.

Com relação ao tipo de cirurgia realizada, foi encontrada uma frequência de evisceração discretamente maior $(52,2 \%)$ que a enucleação (46,7\%). Houve um caso de anoftalmia congênita.

As complicações foram encontradas em 57,1\% dos casos, dos quais a mais frequente foi a extrusão e deiscência (36,5\%), seguida de perto pelas deformidades palpebrais (30,8\%). A maioria das complicações ocorreu tardiamente, mais frequentemente quando a cavidade havia se originado de trauma, seguida de phthisis bulbi (Quadro 1). Dentre as enucleações, a alteração palpebral foi o tipo de complicação mais frequente $(47,8 \%)$, e, dentre as eviscerações, a extrusão foi a mais encontrada (56,0\%).

O implante mais utilizado nas cirurgias foi o de polimetilmetacrilato $(47,2 \%)$ seguido do de polietileno (24,5\%). O tamanho da prótese esférica utilizada variou do diâmetro 15 ao 20, sendo que em $48,2 \%$ dos casos foram introduzidos implantes com tamanho menor ou igual a 17 e, em 51,8\% foram utilizadas esferas de tamanho 18 ao 20.

Foi necessário realizar enxerto de pele na cavidade anoftálmica de $30,0 \%$ dos pacientes e enxerto dermoadiposo, em 32,1\%. A necessidade destes enxertos foi mais notada quando a perda do olho ocorreu pós trauma, seguida de phthisis bulbi. Na maioria dos casos, o aspecto estético (considerando-se o volume e a simetria como critérios para avaliação) foi bom após as cirurgias (Gráfico 3).

Uma parcela significativa dos pacientes $(84,6 \%)$ conseguiu realizar adaptação de prótese externa.

Em relação ao tempo de acompanhamento dos pacientes no serviço, 50,0\% estiveram em acompanhamento por mais de cinco anos e 34,0\% acompanharam por um período de um a cinco anos; o restante perdeu acompanhamento com menos de um ano, a partir da data da primeira consulta, sendo que alguns deles já haviam chegado ao serviço com cirurgias prévias.

\section{DISCUSSÃO}

Estudos retrospectivos pecam pela falta de dados padronizados disponíveis. Entretanto, este tipo de avaliação é muito útil, em especial para nos trazer experiência com determinados procedimentos, técnicas e materiais e muitas vezes a conduta de serviços é alterada em decorrência do que a análise dos casos nos mostra.

Indiscutivelmente o tratamento da cavidade anoftálmica tem se modificado muito nos últimos anos, assim como o perfil demográfico dos pacientes, uma vez que os tratamentos oftalmológicos têm se tornado mais e mais eficientes. Há falta destes dados na literatura nacional, o que nos estimulou a nos lançarmos na revisão dos nossos casos

Neste estudo e em todos os levantamentos que versam sobre cavidade anoftálmica ou sobre trauma ocular, o sexo masculino é apontado como o mais acometido ${ }^{(12)}$, muito provavelmente devido ao fato de o homem estar mais exposto a atividades de risco, a esportes violentos, envolvidos em violência urbana ou acidentes de trânsito(13)

Dentre as causas de perda do olho ou de seu conteúdo, o trauma foi a mais frequente, seguido da phthisis bulbi, o que também foi encontrado em outro estudo(12). Já, a população feminina apresentou principalmente a phthisis bulbi como causa, cabendo ressaltar que a causa do processo que evoluiu para a atrofia ocular pode ser variada e não foi aqui apurada.

Quando se avalia a causa da perda do olho e associação com bilateralidade, o glaucoma e a endoftalmite apareceram como as duas principais doenças, reforçando a import6ancia destas afecções como causa de cegueira.

A avaliação da associação da profissão com a possiblidade de perda da função visual muitas vezes se faz difícil porque são muitas as atividades dos indivíduos, tornando-se difícil categorizá-las. Desta forma, os indivíduos foram classificados de acordo com atividades que envolvem o uso das mãos ou atividades intelectuais. Com este tipo de classificação, foi possível observar que a perda do olho ocorreu mais em sujeitos que utilizam a força física e indivíduos do sexo masculino, o que pode estar associado ao maior porcentual de causas de perda do olho ou de seu conteúdo decorrentes do trauma.

O trauma foi a principal causa de perda ocular em homens de 10 a 40 anos, faixa etária que inclui principalmente indivíduos em idade produtiva e jovens, como concluíram outros autores ${ }^{(12)}$. Em crianças, a causa mais encontrada foi tumor. O trauma, seguido dos tumores, também corresponde a uma parcela significativa na etiologia de cavidades anoftálmicas na infância, o que é justificado pelo fato de que crianças estão mais expostas a processos traumáticos devido a atividades lúdicas que envolvem movimentação física e a acidentes domésticos.

A frequência de eviscerações foi discretamente maior em relação a de enucleações. Atualmente preconiza-se a realização de evisceração ao invés da enucleação sempre que possível pela maior preservação de estruturas orbitárias, exceto em casos de tumores e atrofia bulbar intensa. A ocorrência de extrusões foi maior em eviscerações, comparado a enucleações, resultado semelhante ao de outros ${ }^{(13,14)}$. No nosso serviço, houve um número maior de eviscerações, provavelmente por se acreditar na menor manipulação das estruturas orbitárias com este tipo de procedimento. O maior número de complicações neste grupo de indivíduos pode ter acontecido justamente por se ter realizado maior número de eviscerações do que de enucleções.

As complicações ocorreram em $57,1 \%$ dos casos, dentre as quais as tardias foram as mais encontradas. Apesar de parecer muito grande este número, há que se considerar o longo tempo de seguimento dos pacientes. O achado de complicações nas causas traumáticas também foi maior com relação às outras causas, o que pode estar associado a outras deformidades associadas, como as fraturas ósseas.

A minoria dos indivíduos necessitou de enxertias para expandir os fundos-de-saco. O enxerto de pele ou dermoadiposo estiveram

Quadro 1. Distribuição das complicações após a reconstrução da cavidade anoftálmica segundo época em que ocorreu e a causa de perda do olho ou do seu conteúdo - UNESP, 2010

\begin{tabular}{|c|c|c|c|c|c|c|c|c|}
\hline \multicolumn{9}{|c|}{ Frequência (relativa e absoluta) } \\
\hline \multicolumn{9}{|c|}{ Causa da perda do olho ou de seu conteúdo } \\
\hline Complicação & Glaucoma & Phthisis bulbi & Endoftalmite & Úlcera de córnea & Trauma & Tumor & Outros & Total \\
\hline Precoce (5 casos) & $20,0 \%(1)$ & 0 & $40,0 \%(2)$ & $20,0 \%(1)$ & $20,0 \%(1)$ & 0 & 0 & $100 \%$ \\
\hline Tardia (39 casos) & $5,1 \%(2)$ & $23,08 \%(9)$ & $7,7 \%(3)$ & $5,1 \%(2)$ & $41,0 \%(16)$ & $10,3 \%(4)$ & $7,7 \%(3)$ & $100 \%$ \\
\hline Precoce e tardia (1 caso) & 0 & $100,00 \%(1)$ & 0 & 0 & 0 & 0 & 0 & $100 \%$ \\
\hline Ausente (38 casos) & $10,5 \%(4)$ & $18,40 \%(7)$ & $26,3 \%(10)$ & $5,3 \%(2)$ & $26,3 \%(10)$ & $10,5 \%(4)$ & $2,6 \%(1)$ & $100 \%$ \\
\hline
\end{tabular}




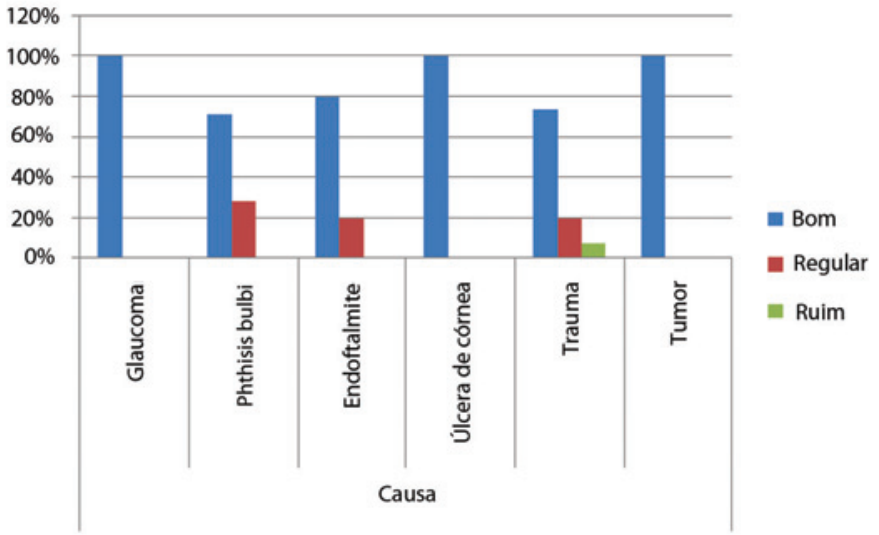

Gráfico 3. Associação do aspecto estético da cavidade anoftálmica de acordo com as causas de perda do olho ou do seu conteúdo - UNESP, 2010.

associados a bom aspecto estético da maioria das cavidades anoftálmicas estudadas.

Na opinião dos autores, portadores de cavidade anoftálmica devem ser acompanhados por toda a vida, uma vez que o quadro é evolutivo e modificações que levam à necessidade de trocas dos implantes ou complicações, podem ser observadas no decorrer do seguimento. Entretanto, muitos abandonam o tratamento, o que também pode influenciar negativamente nas avaliações dos nossos resultados.

\section{CONCLUSÃO}

Os resultados do presente trabalho indicam que o trauma continua sendo a principal causa de perda do olho em nosso meio, ocorrendo principalmente em homens, que trabalham utilizando a força física e as mãos. Com relação à técnica cirúrgica, foi encontrado um porcentual discretamente maior de eviscerações do que de enucleações, com maior número de complicações nas eviscerações, provavelmente por ter sido este tipo de cirurgia o mais realizado. As complicações foram frequentes, muitas aparecendo tardiamente, reforçando a necessidade de acompanhamento prolongado dos pacientes.

\section{REFERÊNCIAS}

1. Soares EJC. Cavidades anoftálmicas. In: Soares EJC, Moura EM, Gonçalves JOR. Cirurgia plástica ocular. São Paulo: Roca; 1997. p.328-30.

2. Silverstone PJ, Beyer-Machule CK, Schaefer DP, Della Rocca RC. Treatment of the anophthalmos and the socket reconstruction. In:Smith BC. Ophthalmic plastic and reconstructive surgery. St. Louis: Mosby; 1987. p.1329-59.

3. Schaefer DP, Della Roca RC. Enucleation. In: Smith BC. Ophthalmic plastic and reconstructive surgery. St. Louis: Mosby; 1987. p.1278-99.

4. Souza Segundo P, SchelliniSA, Padovani CR. Anoftalmia congênita clínica - alterações oculares e sistêmicas associadas. Rev Bras Oftalmol 2006;65(5):269-72.

5. Clauser L, Sarti E, Dallera V, Galiè M. Integrated reconstructive strategies for treating the anophthalmic orbit. J Craniomaxillofac Surg. 2004;32(5):279-90.

6. Siqueira Bisneto O, Barros FS, Schellini SA. Traumas oculopalpebrais no Hospital das Clínicas - Unesp. J Bras Med. 1998:74(6):79-82.

7. Schellini SA, Hoyama E, Padovani CR, Ferreira VL, Roça R. Complicações com uso de esferas não integráveis e integráveis na reconstrução da cavidade anoftálmica. Arq Bras Oftalmol. 2000; 63(3):175-8.

8. Su GW, Yen MT. Current trends in managing the anophthalmic socket after primary enucleation and evisceration. Ophthal Plast Reconstr Surg. 2004;20(4):274-80.

9. Hashimoto M, Rodrigues AC, Silva MRBM, Schellini SA. Cavidade anoftálmica; causas, reconstrução e complicações. Rev Bras Oftalmol. 1994;56(6):73-8.

10. Soll DB. The anophthalmic socket. Ophthalmology. 1982;89(5):407-23.

11. Hashikawa $K$, Terashi $H$, Tahara S. Therapeutic strategy for the triad of acquired anophthalmic orbit. Plast Reconstr Surg. 2007:119(7): 2182-8; discussion 2189-91.

12. Schellini SA, Ichida FK, Padovani CR. Extrusão dos implantes em portadores de cavidade anoftálmica. Arq Bras Oftalmol. 2007;70(5):752-5.

13. Aragaki NG, Inada ET, Teixeira MF, Almeida Júnior GC, Kashiwabuchi LK. Estudo epidemiológico dos traumas oculares graves em um Hospital Universitário de São José do Rio PretoSP. Arq Bras Oftalmol. 2003;66(4):473-6.

14. Zolli CL. Implant extrusion in eviscerations. Ann Ophthalmol. 1988;20(4):127-32,135.

\title{
Envie seu trabalho pelo site do $A B O$
}

\author{
www.scielo.br/abo
}

